\title{
Conception de machines spéciales : methodologie d'élaboration de cahier des charges
}

\author{
Patrick Martin ${ }^{1, a}$, Luc Lossent ${ }^{2},{\text { Laurent } \text { AbT }^{1} \text { et Frédéric Brasset }}^{3}$ \\ 1 Laboratoire de Génie Industriel et Production Mécanique, ENSAM, 4 rue Augustin Fresnel, 57078 Metz Cedex 3, France \\ 2 CRAN, Faculté des sciences, BP 239, 54506 Vandœuvre-lès-Nancy Cedex, France \\ 3 MECANICA, Espace Cormontaigne, BP 10076, 57102 Thionville, France
}

Reçu le 15 janvier 2003, accepté le 16 juin 2003

\begin{abstract}
Résumé - Le caractère spécifique d'une machine spéciale nécessite l'élaboration d'un cahier des charges dans lequel le client exprime son besoin. Il oriente et réalise cette étape en fonction de ses habitudes, sans qu'il n'existe une réelle méthode. En retour, le fournisseur consulté, au travers de son offre, s'engage à satisfaire ce besoin en termes de performances, de coûts, de délais et de qualité. Ainsi, la méthodologie proposée définit une stratégie d'élaboration et de maîtrise du cahier des charges. Elle permet d'assister le donneur d'ordres dans la formalisation de son besoin initial et d'aider le constructeur à traduire ce besoin et à analyser les aspects critiques associés afin d'élaborer un plan d'actions en diminution de risques lui permettant de satisfaire les exigences du client.
\end{abstract}

Mots clés : Conception intégrée / cahier des charges / machine spéciale / analyse de risques

\begin{abstract}
Methodology for specifications in defined machines design. The special characteristics of a defined machine need the elaboration of a specification in which the customer explains his need. He directs and realises this step from force of habit without any real methodology. The consulted supplier through his offer undertakes to satisfy the demand by respecting the performances, the cost, the lead time and the quality. The methodology developed proposes an elaboration and a control strategy of specifications. It assists the principal in the formulation of his initial need and also the constructor in the transformation of the need and in the analyse of the critical aspects. By this way an action plan allowing a lowest level of risks and the satisfaction of the customer demands can be realised.
\end{abstract}

Key words: Concurrent engineering / defined machines / specifications / risk analysis

\section{Introduction}

Les recherches actuelles dans le domaine de la conception [1] s'intéressent essentiellement aux produits de grande série et rarement aux produits unitaires tels que le système de production lui-même ou la machine spéciale [2-4]. D'autre part la phase amont d'élaboration du cahier des charges est également peu étudiée malgré les risques et incertitudes, l'utilisation des normes [5] restant souvent insuffisante.

Cet article se place dans ce cadre suite à une étude menée en partenariat avec le centre de transfert de technologie pour les industries mécaniques lorrain : MECANICA. Il porte sur la conception de machines spéciales, donc des produits unitaires dédiés à un type d'application ne relevant pas nécessairement de l'industrie mécanique.

\footnotetext{
a Auteur correspondant : patrick.martin@metz.ensam.fr
}

Le concepteur n'est pas forcément familier du procédé et des contraintes associées. Il est amené à répondre rapidement à des appels d'offre à partir de cahiers des charges plus ou moins précis ou complets. Compte tenu de l'incertitude sur l'éventualité de réaliser effectivement la machine, le constructeur est amené à réaliser le devis à partir d'une étude grossière en prenant un certain nombre d'hypothèses; de plus il prend des risques techniques et économiques. Les machines comportent une partie opérative et une partie commande, nous nous intéresserons essentiellement au premier aspect.

MECANICA a lancé une action visant à aider les constructeurs dans cette tâche. Tout d'abord une enquête réalisée auprès de différentes entreprises lorraines (fabricants et donneurs d'ordre), a montré que des échecs s'étaient produits à la suite d'erreurs d'estimation sur les performances attendues, elle a également conclu à 
une demande d'amélioration de la réalisation de cahier des charges de machines spéciales. La formalisation sous forme de cahier des charges ne suffit pas à garantir le succès d'un projet, ni même à quantifier les risques qui lui sont associés. Pour un constructeur de machines spéciales, il est primordial de pouvoir maîtriser le projet qu'il se voit confier, car il porte seul la responsabilité de ses actes et garantit le résultat final. Il lui appartient donc de savoir identifier les risques potentiels et de déclencher les modes d'actions appropriés afin de satisfaire le besoin d'un point de vue qualitatif, et de rester parfaitement maître du projet en termes de coûts et de délais. Un audit a été mené auprès d'entreprises concepteurs et donneurs d'ordre afin de dresser un « état des lieux », aussi bien sur leurs habitudes de travail, sur les moyens et les ressources dont elles disposent que sur les problématiques auxquelles elles se trouvent confrontées. Ceci nous a conduit à bâtir une stratégie d'élaboration et de maîtrise du cahier des charges [6] qui fait l'objet de cet article.

La méthode ainsi proposée a pour objectif de permettre :

- au donneur d'ordres : de décrire leurs besoins au travers d'un cahier des charges initial puis de leurs faciliter la tâche de validation d'une offre grâce à un modèle structuré et formalisé;

- au constructeur : de l'aider à traduire ce besoin et à identifier, quantifier et diminuer le taux de criticité lié à l'acceptation de l'affaire.

\section{Quelques définitions}

\section{Machine spéciale :}

La machine spéciale est une machine répondant à un besoin spécifique du client qui ne peut trouver satisfaction dans ce qui est habituellement vendu sur catalogue. Elle est fabriquée sur mesure, à l'unité ou en petit nombre. Par conséquent, elle est, par définition, opposée à la machine standard.

Nous avons nommé la méthodologie proposée, SEMCaDec, pour Stratégie d'Élaboration et de Maîtrise du Cahier des charges. Rappelons tout d'abord les définitions associées à ces différents termes, définitions qui permettent de préciser le contexte du travail réalisé :

- stratégie : art de diriger un ensemble de dispositions pour atteindre un but;

- élaboration : préparation et formalisation d'un plan d'actions pour atteindre un objectif;

- maîtrise : domination incontestée dans l'accomplissement d'un acte;

- cahier des charges : document par lequel le demandeur exprime son besoin d'un point de vue fonctionnel (en termes de fonctions et de contraintes) et technique (en termes de performances du moyen).

Tout au long des discussions client-fournisseur, le cahier des charges évolue et a différents états, il est donc important de bien préciser ces différentes versions.
Ces états du cahier des charges ne forment en réalité qu'un seul et même document basé sur un échange de données et d'informations entre le client et le constructeur.

1. CdCV0 : Cahier des Charges Version 0 (réalisé par le client).

Document dans lequel le client exprime son besoin, avec les données dont il dispose, et ses objectifs en termes de performances, de coûts et de délais.

2. $\mathrm{CdCF}$ : Cahier des Charges fonctionnel (réalisé par le client, complété par le fournisseur).

Document dans lequel le client exprime son besoin en termes de fonctions de service et de contraintes. Pour chacune d'elles sont définis des critères d'appréciation et leurs niveaux. Chacun de ces niveaux doit être assorti d'une flexibilité [NFX 50-150].

L'Analyse fonctionnelle contenue dans le CdCF vient compléter les données initiales du CdCV0 et peut être à la charge du constructeur.

3. CdCT : Cahier des Charges Technique (réalisé par le fournisseur en tout ou partie, éventuellement par le client).

Document par lequel est décrit le principe retenu à l'issue d'une phase d'étude de faisabilité ou le détail d'une solution technique à une étape donnée du projet.

4. CdCC : Cahier des Charges Constructeur/ Concepteur (réalisé par le constructeur, permet de formaliser son offre).

Document établi à partir de concepts directeurs validés et vérifiant la trilogie besoin, stratégie et veille de l'entreprise. Le CDCC fige le design, la technologie et la performance du produit.

5. CdCV1 : Cahier des Charges Version 1 (réalisé par le client, permet de formaliser un document contractuel d'entrée en partenariat).

Le CdCV1 contient toutes les données évoquées depuis le CdCV0 et reprend l'ensemble des données du fournisseur dont l'offre aura été retenue. Il a une valeur contractuelle et permet d'établir un contrat de commande client.

6. DTM : Dossier Technique Machine (réalisé par le constructeur).

Ce dossier doit être finalisé et remis au client au moment de la réception finale de la machine. Il décrit la présentation de l'équipement (présentation du projet : CdCV1, demandes d'études, offre du fournisseur, spécifications techniques, etc.) ainsi que les instructions d'installation (dossiers de plans, spécifications techniques), de mise en service et de maintenance (instructions de mise en service et de maintenance, conformité à la réglementation). 


\section{Présentation de la méthodologie}

Le projet est découpé en quatre métaphases constituées de huit phases au total (Fig. 1) et correspondant chacune à une étape de création du cahier des charges $(\mathrm{CdC})$.

La métaphase 1 : expression du besoin, permet d'élaborer un document dans lequel est exprimé le besoin initial au travers du CdCV0 ou du CdCF, en vue de déclencher un appel d'offre. Cette métaphase est découpées en 4 phases : identification, perception, description et analyse fonctionnelle.

La métaphase 2 : traduction du besoin, permet de traduire techniquement le besoin par l'intermédiaire du CdCT, dans le but de répondre à l'appel d'offre en termes de propositions de solutions techniques. C'est la conception.

La métaphase 3 : analyse du plan d'actions, permet d'aboutir au CdCC après avoir mené une analyse des risques d'application liés au projet. Une fois son plan d'action défini, le constructeur rédige son offre ou la décline. Il réalise alors une proposition.

La métaphase 4 : entrée en partenariat, permet d'analyser les différentes solutions techniques et de comparer les offres des fournisseurs de manière à retenir un prestataire de services. Cette métaphase aboutit au CdCV1 négocié, permettant de formaliser les données et les informations nécessaires à la rédaction du contrat d'entrée en partenariat. Elle permet d'élaborer le cahier des charges depuis son état initial jusqu'à son état négocié et validé par le client. Le cahier des charges $(\mathrm{CdC})$ fait alors office de document contractuel, donnant lieu à la signature du contrat de la part des deux parties. Elle s'inscrit au sein du management de projet dans l'étape nommée « entrée en partenariat $»$. Elle contient la phase de validation.

De plus des dates jalons ont été définies, elles correspondent à la date limite fixée à l'issue de laquelle une action doit aboutir pour atteindre un objectif donné, permettant ainsi le déclenchement de l'action suivante. Par ailleurs, ce jalonnement du projet permet de mieux maîtriser les éventuelles dérives afin de veiller au respect du délai global.

Description des dates jalons : X0, X1, X2, X3 : dates jalon client.

- X0 : correspond à la date de démarrage du projet du point de vue du client, dès l'instant où l'étude d'opportunité est validée et que l'autorisation de dépense est signée.

- X1 : date à laquelle le contrat d'entrée en partenariat est signé des deux parties et réceptionné par le client.

- X2 : date pour la réception provisoire par le client chez le fournisseur avant expédition.

- X3 : correspond à la clôture du projet. J0, J1, J2, J3 : dates jalon constructeur.

- J0 : date à laquelle le sous-traitant reçoit le document et fige le délai dont il dispose pour répondre à l'appel d'offre.

- J1 : date de remise de l'offre.
- J2 : date à laquelle le fournisseur remet le dossier complet au client pour approbation avant réalisation.

Le flux des données et les actions associées au cours des phases sont présentées en figure 1 .

La méthode fait apparaître des invariants dans les différentes phases d'identification, de perception, de description, d'analyse fonctionnelle, de conception, d'analyse du plan d'actions, de proposition et de validation, qui nous permettent de limiter la complexité des objets à créer dans la phase d'informatisation afin de la rendre rapidement exploitable sur le terrain. Ces invariants sont : phase, sous phase, paragraphe.

Pour le client, les métaphases permettent de formaliser son besoin et de le guider pendant l'élaboration du cahier des charges permettant la réalisation de l'appel d'offre. Pour cela la granulométrie de l'analyse a été poussée afin de répondre à l'attente des industriels. Pour le fournisseur, cette méthodologie lui permet de traduire le cahier des charges fourni afin de bien s'assurer de la compréhension des différents aspects techniques, économiques et juridiques. L'échange d'informations provoqué entre client et fournisseur ainsi que la traçabilité intégrée dans la méthodologie entraîne donc la «co-élaboration »du Cahier des Charges.

La circulation des informations client-fournisseur se transforme en communication par leur validation autour d'une méthode commune et prépare l'entrée en partenariat. La complexité de co-rédaction du cahier des charges de machines spéciales qui entraîne les deux parties d'une part à gérer un flux important de données, d'autre part à les compléter ou les préciser est directement prise en compte dans cette méthodologie. Ainsi son utilisation permet la structuration de l'information technique, une comparaison rigoureuse de plusieurs alternatives, et une prise de décisions à partir de ces informations ou de détecter celles sur lesquelles le risque sera plus important.

Le tableau comparatif 1 reprend les 8 phases de SEMCaDeC et les compare aux 7 étapes de la démarche d'analyse de la valeur, afin de montrer, suivant un référentiel connu, quel est l'objectif de chacune de ces phases.

\section{Analyse de risque}

Nous allons détailler la démarche associée contenue dans la métaphase 3 et notamment dans la définition des prestations. Cette phase n'existe pas dans la démarche d'analyse de la valeur. Nous trouvons une approche similaire dans la méthode SPEC [7-9]. Dans l'analyse du plan d'actions, nous avons proposé 5 《 familles »d'aspects critiques (technique, délais, contraintes, information, matériel et logistiques) que nous avons découpé en 5 paragraphes.

Nous cherchons les risques potentiels en les identifiant et les hiérarchisant. Nous procédons à une estimation de la gravité du risque et de la probabilité d'apparition du risque :

- gravité de l'impact qu'un risque peut avoir sur le déroulement d'un plan d'actions ; 


\begin{tabular}{|c|c|c|c|c|c|c|c|}
\hline \multirow{2}{*}{ Etapes du projet } & \multirow{2}{*}{ Objectifs } & \multirow{2}{*}{ Etat du CdC } & \multirow{2}{*}{ Phases } & \multirow{2}{*}{ Actions } & \multirow{2}{*}{ Document sortant } & \multicolumn{2}{|c|}{ Responsables } \\
\hline & & & & & & Client & Constructeur \\
\hline $\begin{array}{c}\text { ETUDE } \\
\text { D'OPPORTUNITE }\end{array}$ & $\begin{array}{c}\text { Valider le besoin de } \\
\text { l'étude par rapport aux } \\
\text { besoins du marché et à } \\
\text { la stratégie de } \\
\text { l'entreprise }\end{array}$ & & & & $\begin{array}{l}\text { Rapport d'étude } \\
\text { d'opportunité } \\
\text { Note interne client }\end{array}$ & $\begin{array}{l}\text { Commandi- } \\
\text { taire / Equipe } \\
\text { de direction }\end{array}$ & \\
\hline \multirow[t]{3}{*}{$\begin{array}{c}\text { ENTREE EN } \\
\text { PARTENARIAT }\end{array}$} & $\begin{array}{l}\text { Exprimer le besoin } \\
\text { initial et décrire le } \\
\text { contexte (impératifs, } \\
\text { contraintes, etc) }\end{array}$ & Initial & $\begin{array}{l}\text { IDENTIFICATION } \\
\text { PERCEPTION } \\
\text { DESCRIPTION }\end{array}$ & $\begin{array}{c}\text { Initier le projet } \\
\text { Exprimer le besoin identifié } \\
\text { Décrire l'existant et le travail } \\
\text { Etre en mesure de rédiger un } \\
\text { appel d'offre }\end{array}$ & $\begin{array}{l}\text { Etude de faisabilité } \\
\text { CdC V0 initial }\end{array}$ & $\begin{array}{c}\text { Rédacteur du } \\
\text { CdC V0 }\end{array}$ & \\
\hline & Analyser les fonctions & $\begin{array}{l}\text { Analysé } \\
\text { (niveau 1) }\end{array}$ & $\begin{array}{l}\text { IDENTIFICATION } \\
\text { PERCEPTION } \\
\text { DESCRIPTION } \\
\text { ANALYSE } \\
\text { FONCTIONNELLE }\end{array}$ & $\begin{array}{c}\text { Compléter et renseigner (le } \\
\text { constructeur) } \\
\text { Traduire le besoin client en } \\
\text { fonctions } \\
\text { Raisonner en termes de } \\
\text { fonctions et non de solutions, } \\
\text { déclencher une Analyse de la } \\
\text { Valeur }\end{array}$ & $\begin{array}{c}\text { Matrice des } \\
\text { interactions } \\
\text { CdCF (Cahier des } \\
\text { Charges Fonctionnel) }\end{array}$ & & 4 \\
\hline & $\begin{array}{l}\text { Proposer, choisir et } \\
\text { valider des solutions } \\
\text { technologiques }\end{array}$ & $\begin{array}{l}\text { Analysé } \\
\text { (niveau 2) }\end{array}$ & $\begin{array}{l}\text { IDENTIFICATION } \\
\text { PERCEPTION } \\
\text { DESCRIPTION } \\
\text { ANALYSE } \\
\text { FONCTIONNELLE } \\
\text { CONCEPTION }\end{array}$ & $\begin{array}{l}\text { Compléter l'information, enrichir } \\
\text { les données } \\
\text { Déterminer les concepts } \\
\text { Etre en mesure de valider } \\
\text { l'aspect technique / faisabilité }\end{array}$ & $\begin{array}{l}\text { CdCT (Cahier des } \\
\text { Charges Technique) }\end{array}$ & & $\begin{array}{c}\text { Chef de } \\
\text { projet } \\
\text { constructeur }\end{array}$ \\
\hline \multirow{2}{*}{ Etapes du projet } & \multirow{2}{*}{ Objectifs } & \multirow{2}{*}{ Etat du CdC } & \multirow{2}{*}{ Phases } & \multirow{2}{*}{ Actions } & \multirow{2}{*}{ Document sortant } & \multicolumn{2}{|c|}{ Responsables } \\
\hline & & & & & & Client & Constructeur \\
\hline \multirow[t]{4}{*}{$\begin{array}{c}\text { ENTREE EN } \\
\text { PARTENARIAT }\end{array}$} & $\begin{array}{c}\text { Analyser les risques et } \\
\text { mener des actions en } \\
\text { diminution de risques }\end{array}$ & $\begin{array}{l}\text { Analysé } \\
\text { (niveau 3) }\end{array}$ & $\begin{array}{l}\text { IDENTIFICATION } \\
\text { PERCEPTION } \\
\text { DESCRIPTION } \\
\text { ANALYSE } \\
\text { FONCTIONNELLE } \\
\text { CONCEPTION } \\
\text { ANALYSE DU } \\
\text { PLAN D'ACTIONS }\end{array}$ & $\begin{array}{l}\text { Analyser et estimer les risques } \\
\text { associés au projet } \\
\text { Etablir un plan d'actions en } \\
\text { proposant des modes d'actions } \\
\text { en diminution de risques }\end{array}$ & $\begin{array}{c}\text { ARA / ADEQUA } \\
\text { ("AMDEC pour CdC") }\end{array}$ & & \\
\hline & $\begin{array}{l}\text { Décider d'un plan } \\
\text { d'actions }\end{array}$ & Renseigné & $\begin{array}{l}\text { IDENTIFICATION } \\
\text { PERCEPTION } \\
\text { DESCRIPTION } \\
\text { AF } \\
\text { CONCEPTION } \\
\text { PLAN D'ACTIONS } \\
\text { PROPOSITION }\end{array}$ & $\begin{array}{l}\text { Soumettre au client une } \\
\text { proposition de prestation } \\
\text { Formaliser les conditions } \\
\text { d'entrée en partenariat }\end{array}$ & $\begin{array}{c}\text { CdCC (Cahier des } \\
\text { Charges Constructeur) } \\
\text { Offre succincte ou } \\
\text { détaillée }\end{array}$ & & $\begin{array}{c}\text { Chef de } \\
\text { projet } \\
\text { constructeur }\end{array}$ \\
\hline & \multirow[t]{2}{*}{$\begin{array}{c}\text { Valider l'entrée en } \\
\text { partenariat }\end{array}$} & Négocié & \multirow[t]{2}{*}{$\begin{array}{l}\text { IDENTIFICATION } \\
\text { PERCEPTION } \\
\text { DESCRIPTION } \\
\text { AF } \\
\text { CONCEPTION } \\
/ / / / / / / / / / / / / / / / / / / / / \\
\text { PROPOSITION } \\
\text { VALIDATION }\end{array}$} & \multirow[t]{2}{*}{$\begin{array}{l}\text { (Plan d'actions masqué dans } \\
\text { la vue "méthodologie client") } \\
\text { Valider l'entrée en partenariat } \\
\text { Etablir un contrat de commande } \\
\text { (à partir de l'offre retenue) }\end{array}$} & $\begin{array}{c}\text { CdC V1 validé } \\
\text { Contrat de commande }\end{array}$ & $\begin{array}{c}\text { Chef de } \\
\text { projet client }\end{array}$ & \\
\hline & & Jalon "X1" & & & & & \\
\hline
\end{tabular}

Fig. 1. Phases, actions et documents associés. 
Tableau 1. Méthodologie SEMCaDeC inscrite dans une démarche Analyse de la Valeur.

\begin{tabular}{|c|c|c|c|}
\hline $\mathrm{AV}$ & \multicolumn{2}{|c|}{ SEMCaDeC } & Les atouts majeurs de SEMCaDeC \\
\hline $\begin{array}{l}\text { Orientation } \\
\text { de } \\
\text { l'action }\end{array}$ & \multirow{4}{*}{ 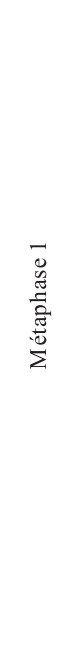 } & $\begin{array}{l}\text { Phase } \\
\text { d'IDENTIFICATION }\end{array}$ & $\begin{array}{l}\text { - Mentionne nominativement chaque acteur du projet et ses responsabilités, } \\
\text { - Décompose le projet en sous-parties afin de faciliter l'étude et l'analyse des } \\
\text { points critiques. }\end{array}$ \\
\hline \multirow{2}{*}{$\begin{array}{l}\text { Recherche } \\
\text { de } \\
\text { l'information }\end{array}$} & & $\begin{array}{l}\text { Phase de } \\
\text { PERCEPTION }\end{array}$ & $\begin{array}{l}\text { - Permet de revalider les buts et causes du projet, } \\
\text { - Synthétise le rapport d'étude d'opportunité, } \\
\text { - Inventorie les performances souhaitées et pro-pose un CR et une analyse } \\
\text { exhaustive de l'existant (d'autant plus important que la base de données } \\
\text { s'enrichit). }\end{array}$ \\
\hline & & $\begin{array}{l}\text { Phase de } \\
\text { DESCRIPTION }\end{array}$ & $\begin{array}{l}\text { - Jalonne le projet afin de maîtriser les délais, } \\
\text { - Interaction avec le planning prévisionnel sur MS Project avec répercussion des } \\
\text { modifications, } \\
\text { - Prend en compte les normes et les standards en vigueur, } \\
\text { - Formate l'offre attendue par le client. }\end{array}$ \\
\hline $\begin{array}{l}\text { Analyse des fonctions et } \\
\text { des coûts }\end{array}$ & & $\begin{array}{l}\text { Phase } \\
\text { d'ANALYSE } \\
\text { FONCTIONNELLE }\end{array}$ & - Orientation dans la démarche d'Analyse Fonctionnelle. \\
\hline Recherche de solutions & \multirow{2}{*}{ 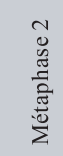 } & \multirow{2}{*}{$\begin{array}{l}\text { Phase de } \\
\text { CONCEPTION }\end{array}$} & \multirow{2}{*}{$\begin{array}{l}\text { - Recherche de concepts facilitée grâce à une recherche par mots clés dans la } \\
\text { base de données, } \\
\text { - Appels à outils tels que TRIZ, } \\
\text { - Méthodologies QFD et ADO. }\end{array}$} \\
\hline Evaluation des solutions & & & \\
\hline $\begin{array}{l}\text { (Cette phase n'existe pas } \\
\text { dans la démarche A V) }\end{array}$ & \multirow{2}{*}{ 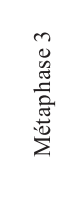 } & $\begin{array}{l}\text { Phase } \\
\text { d'ANALYSE du } \\
\text { PLAN D'ACTIONS }\end{array}$ & - Ajout d'une étape capitale : ARA (Analyse des Risques d'Application) \\
\hline Proposition de choix & & $\begin{array}{l}\text { Phase de } \\
\text { PROPOSITION }\end{array}$ & $\begin{array}{l}\text { - Formalisation de l'offre intégrant de manière « automatique» les conclusions } \\
\text { du plan d'actions }\end{array}$ \\
\hline Bilan / Choix du décideur & 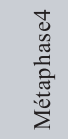 & $\begin{array}{l}\text { Phase de } \\
\text { VALIDATION }\end{array}$ & $\begin{array}{l}\text { - Grandement facilitée par la formalisation de l'offre sous forme standardisée et } \\
\text { grâce à l'outil ADO (Analyse de Décision Opérationnelle). }\end{array}$ \\
\hline
\end{tabular}

- probabilité que la cause, origine d'un risque, ne survienne.

En graduant la gravité et la probabilité de 0 à 10 (Fig. 2), nous obtenons des modes d'actions préventives qui agissent sur la probabilité, des modes d'actions de secours qui agissent sur la gravité et des modes d'actions de déclenchement qui agissent sur les deux. Nous obtenons le taux de criticité (comme dans une étude AMDEC) par le produit «GRAVITE X PROBABILITE » en utilisant ces échelles de mesure. Les taux de criticité les plus élevés sont pris en compte, la recherche des causes permet d'enclencher les modes d'actions. Les modes d'actions pour la diminution de risques sont guidés par l'utilisation de ce critère. Ces modes sont ensuite réinjectés dans le plan d'application et le taux de criticité résiduel est recalculé. Cette phase permet au constructeur de rédiger une offre en ayant optimisé son plan d'actions grâce à des modes d'actions en diminution de risques.

\section{Modélisation de SEMCaDeC}

\subsection{Méthodologie}

Afin de permettre la structuration de l'ensemble des données et des actions, l'architecture de la méthodologie a été modélisée à l'aide d'OBJETS TECHNIQUES constitués de données, de liens entre ces objets et formant une vue appelée « MÉTHODOLOGIE » (Fig. 3).

Les liens sont : «caractérisé par», « renseigné par », «composé de », «supporté par» et «documenté par ». Les OBJETS sont ceux décrits dans les quatre métaphases et ceux nécessaires pour documenter et renseigner sur les outils et les méthodes utilisés, ainsi que des objets paramétrables par l'utilisateur. Ils possédent de 3 à 7 CHAMPS : PROJET, PHASE, ÉTIQUETTE, SOUS-PHASE, PARAGRAPHE, CHAMPS, OUTILS, MÉTHODOLOGIE et DOCUMENT. La vue comprend les 8 phases détaillées à la figure 1 . 


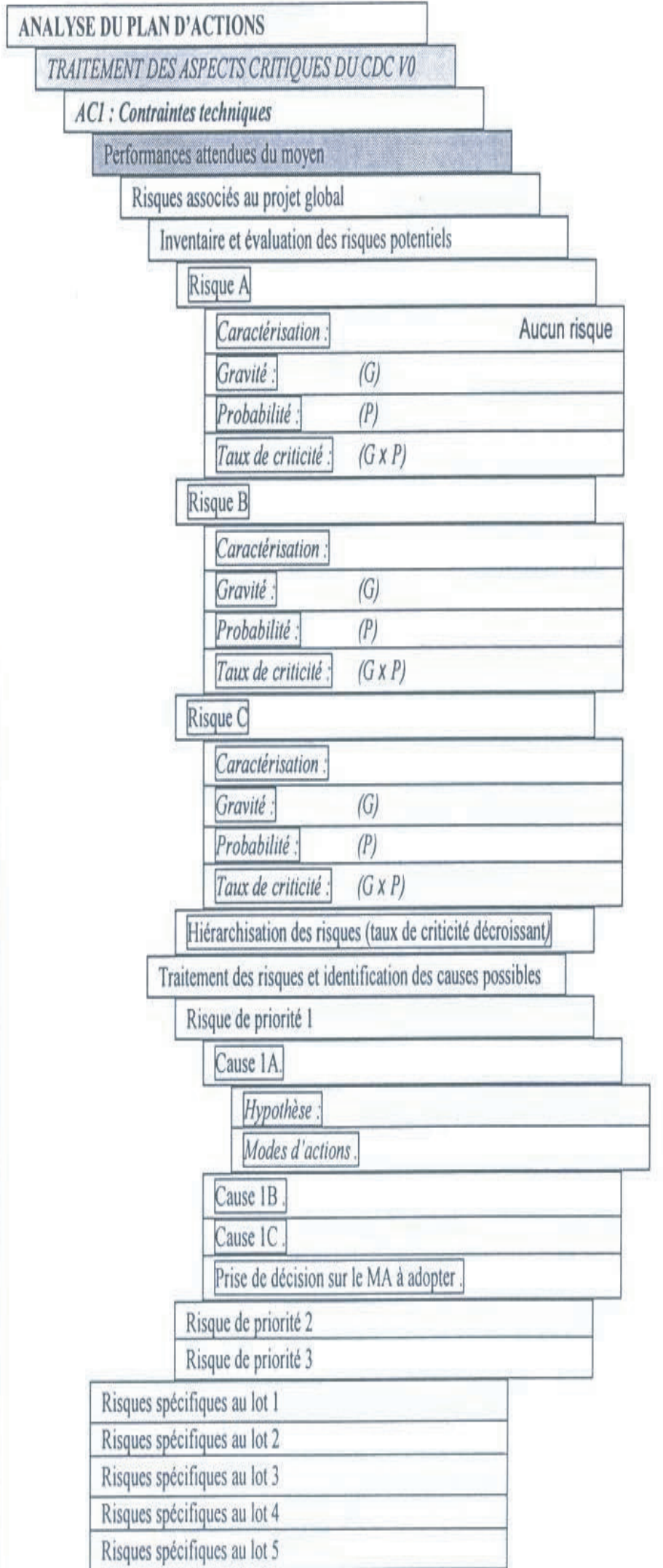

\begin{tabular}{|l|}
\hline Risques potentiels \\
\hline Performances trop spécifiques. \\
\hline Incompatibilités produit / process. \\
\hline Analyse longue et coûteuse. \\
\hline Ne pas répondre ou répondre partiellement au besoin. \\
\hline Mauvaise maitrise du projet. \\
\hline Engager sa responsabilité sur des données non maitrisées. \\
\hline Ne pas tenir ses engagements en ce qui concerne les délais. \\
\hline Ne pas tenir ses engagements concernant le coùt global estimé. \\
\hline Répondre en termes de solutions et non en termes de fonctions. \\
\hline Aucun risgue. \\
\hline Autre (à saisir) \\
\hline
\end{tabular}

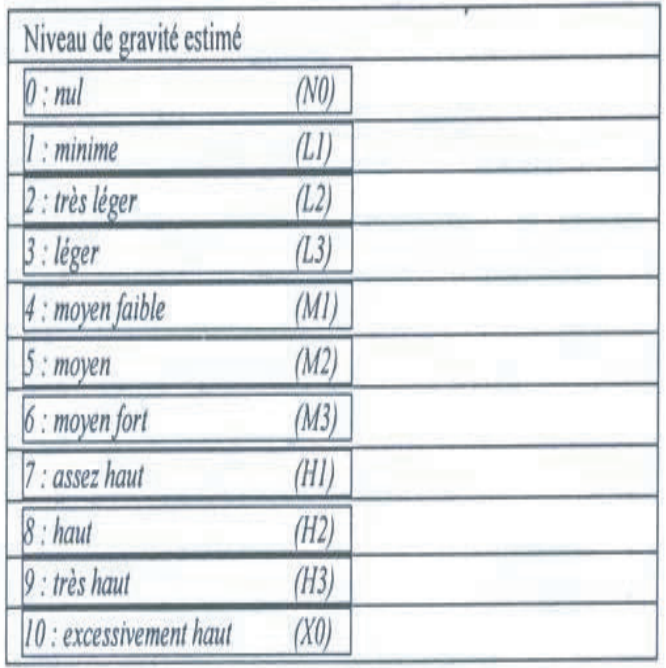

\begin{tabular}{|c|c|c|}
\hline \multicolumn{3}{|c|}{ Niveau d'occurence (probabilité d'apparition du risque) } \\
\hline $0: \mathrm{mul}$ & (NO) & \\
\hline 1:minime & (LI) & \\
\hline 2: très mince & $(L 2)$ & \\
\hline 3: mince & $(L 3)$ & \\
\hline 4:moyen faible & (MI) & \\
\hline 5:moyen & (M2) & \\
\hline 6: moyen fort & (M3) & \\
\hline 7: assez fort & (HI) & \\
\hline 8:fort & (H2) & \\
\hline 9:très fort & $(H 3)$ & \\
\hline I0: excessivement fort & $(X 0)$ & \\
\hline
\end{tabular}

Fig. 2. Traitement des aspects critiques de la phase d'analyse du plan d'action (métaphase 3). 


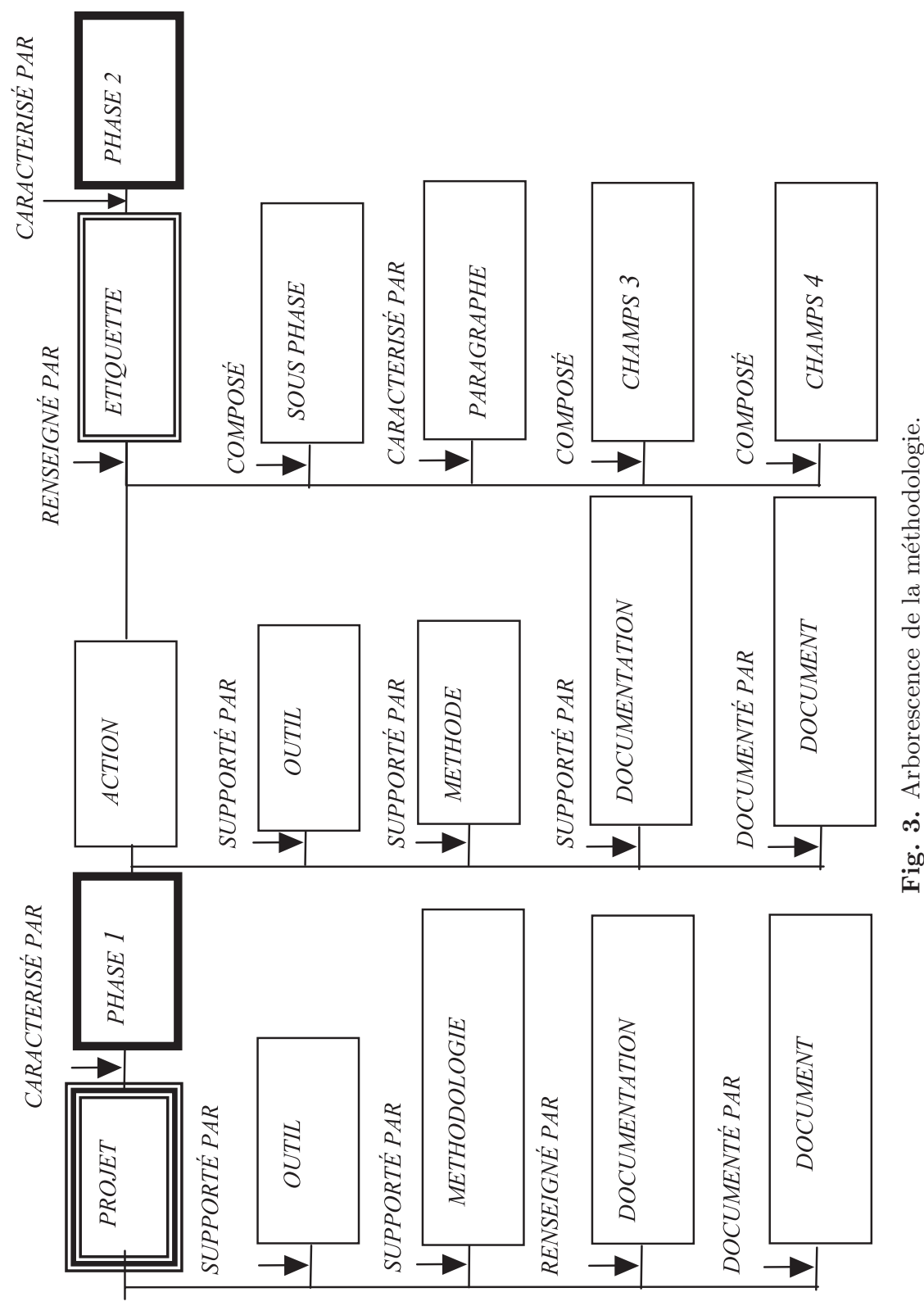


Le PROJET est la tête de l'arborescence. Chaque PHASE est décomposée en ACTION renseignée par une ou plusieurs ÉTIQUETTES. Une ACTION est supportée par un ou plusieurs OUTILS et MÉTHODES. Le PROJET et les PHASES sont renseignés en cours de réalisation par de la DOCUMENTATION puis en final documenté par DOCUMENT. Une ÉTIQUETTE est composée de SOUS-PHASES caractérisé par un PARAGRAPHE composé de $« \mathrm{n} »$ niveaux qui sont des OBJETS de 3 à 7 champs suivant les besoins (contexte du projet, type de phase ...) et donc choisis par l'utilisateur. Ce découpage a permis pour la maquette de validation de la méthodologie de limiter le nombre d'objets techniques. La PHASE 2 est constituée de la même manière.

Les objets CHAMPS sont utilisés notamment pour la description des lots.

Nous présentons à la figure 4 la phase de traduction du besoin et d'entrée en partenariat, les résultats de la maquette de modélisation ne sont vus que par les concepteurs de la méthodologie, l'utilisateur de la méthodologie a une interface du type formulaire de système de gestion de base de données.

La création de la méthodologie SEMCaDeC dans le système de gestion de configuration, nécessite de travailler par étapes :

- l'analyse et la validation des invariants a permis le recensement du nombre, du type et de la nature des objets nécessaires à la modélisation;

- le paramétrage des objets techniques, c'est-à-dire leur création et la définition de leurs attributs, ainsi que la création des liens et des vues;

- création des tables, cette étape et la précédente constituent le travail de paramétrage des objets techniques et de leur table associée dans la base de données;

- création de la base «méthodologie» à partir de fichiers d'aide constitués des invariants du $\mathrm{CdC}$ et importés via Excel dans la base, les invariants sont répartis selon le type d'objet technique qu'ils représentent (phases, étiquettes, sous-phases, etc.). Nous affectons ainsi nos paramètres invariants dans les tables de la base de données, de telle manière à pouvoir créer l'architecture de la méthodologie;

- création de l'arborescence sous le logiciel de gestion de bases de données techniques, en affectant aux différents objets leur emplacement et leur fonction à l'aide des liens. Avant de pouvoir créer l'arborescence, il est nécessaire d'indexer chaque objet technique, c'est-à-dire de renseigner les paramètres d'indexation (ou champs) obligatoires (et éventuellement facultatifs) qui leur ont été affectés lors du paramétrage de manière à les caractériser et à les rendre actifs. C'est cette étape qui permet de procéder ensuite à des comparaisons d'indice à indice, de date à date, ou d'effectuer des confrontations en fonction des fournisseurs, ou de tout autre paramètre susceptible de représenter un critère de recherche ou de comparaison.

\subsection{Application}

Une maquette informatique basée sur un logiciel de gestion de configuration a été développée (Fig. 5) et a permis de valider la méthode sur une application pour l'industrie automobile.

Le cahier des charges qui a été remis au sous-traitant concerne la modification et l'intégration d'un poste de serrage automatique sur une ligne d'assemblage de motorisations diesel.

Le contenu du cahier des charges, nommé « cahier des charges pour consultation », est aussi détaillé que pouvait le présager la simple lecture du titre : «Intégration du poste de serrage du support moteur XX récupéré. Poste à adapter pour le serrage de 4 vis capotage inférieur et de 6 vis support moteur. Pour les motorisations YY dans l'atelier de montage $\mathrm{AA} »$. Il fait par ailleurs référence à d'autres documents, qui eux-mêmes font appels à des normes AFNOR ou à des normes et standards internes.

Par conséquent, notre volonté a été de synthétiser dans un seul document, toutes les informations nécessaires et suffisantes. Nous avons cherché à structurer les paragraphes de façon à éviter de réitérer la même idée dans deux paragraphes différents ou d'évoquer une même notion en utilisant un vocabulaire différent et non explicité. La structure arborescente obtenue qui présente les métaphases en renseignant les invariants à partir des données relatives au poste de serrage est relativement volumineux. Un exemple est présenté figure 6 .

La structure arborescente, bien que peu conviviale, a eu l'avantage d'être explicite au moment de l'expression du besoin vis-à-vis du sous-traitant. Il n'a nullement la prétention de représenter le document qui sera remis aux fournisseurs consultés, puisque ce dernier sera, à terme, généré par le SGDT au format HTML et mis en forme suivant une présentation conventionnelle.

\subsection{Autre développement}

D'autre part un prototype portant principalement sur la partie élaboration de devis a été développé en utilisant la méthodologie présentée, sous le logiciel ACCESS de Microsoft Office. Celui-ci doit s'intégrer dans l'offre logicielle de MECANICA constituée d'un ensemble d'outils d'aide à la conception de machine spéciale accessible via Internet.

\section{Conclusion}

La méthodologie de maîtrise de cahier des charges développée permet :

- de générer de manière simple et réactive, une configuration conforme aux exigences contractuelles et aux spécifications en prenant en compte :

- la configuration de référence;

- les demandes de dérogations et leurs statuts;

- les ordres de modifications et leurs états ; 


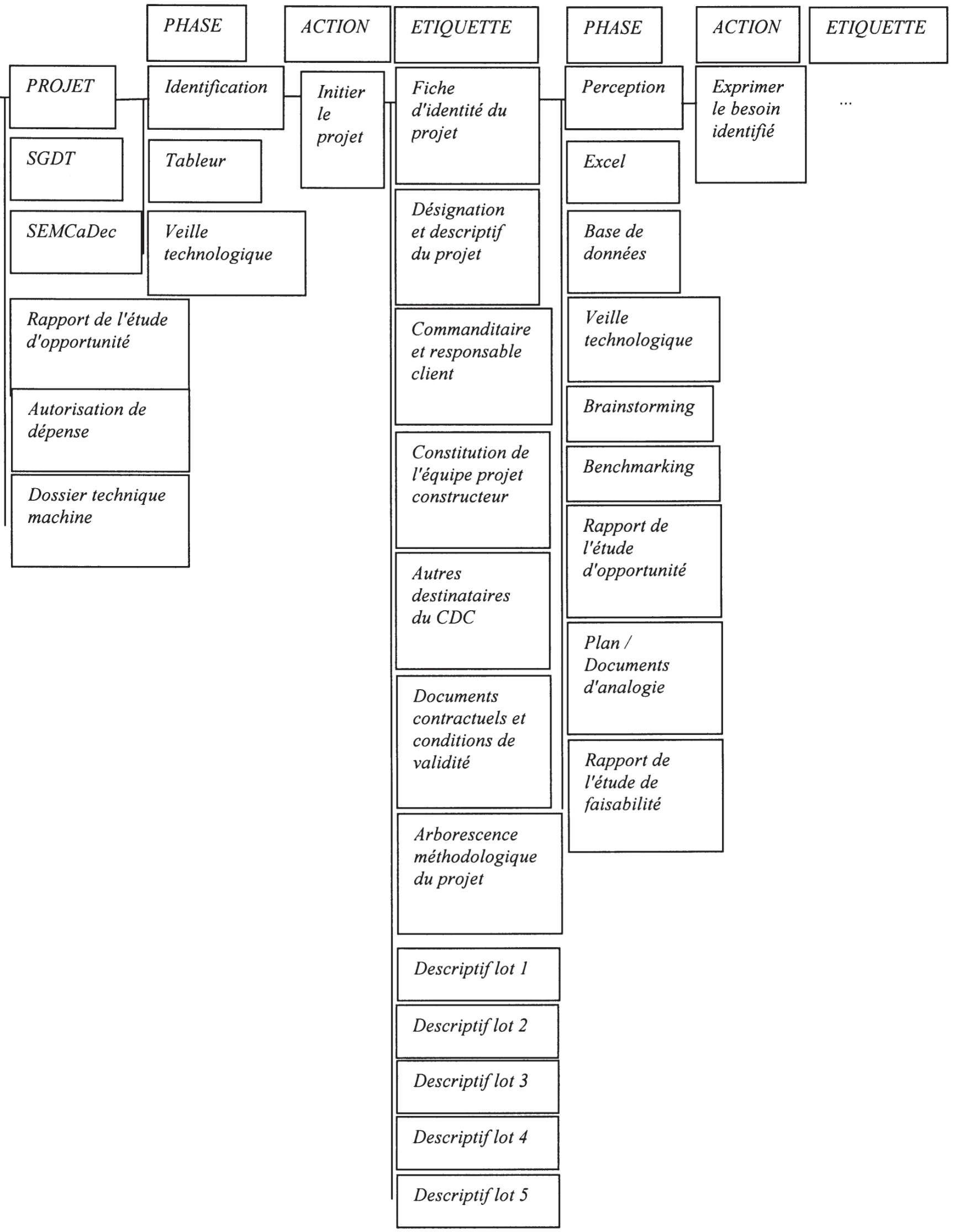

Fig. 4. Phases de perception et d'identification du besoin. 


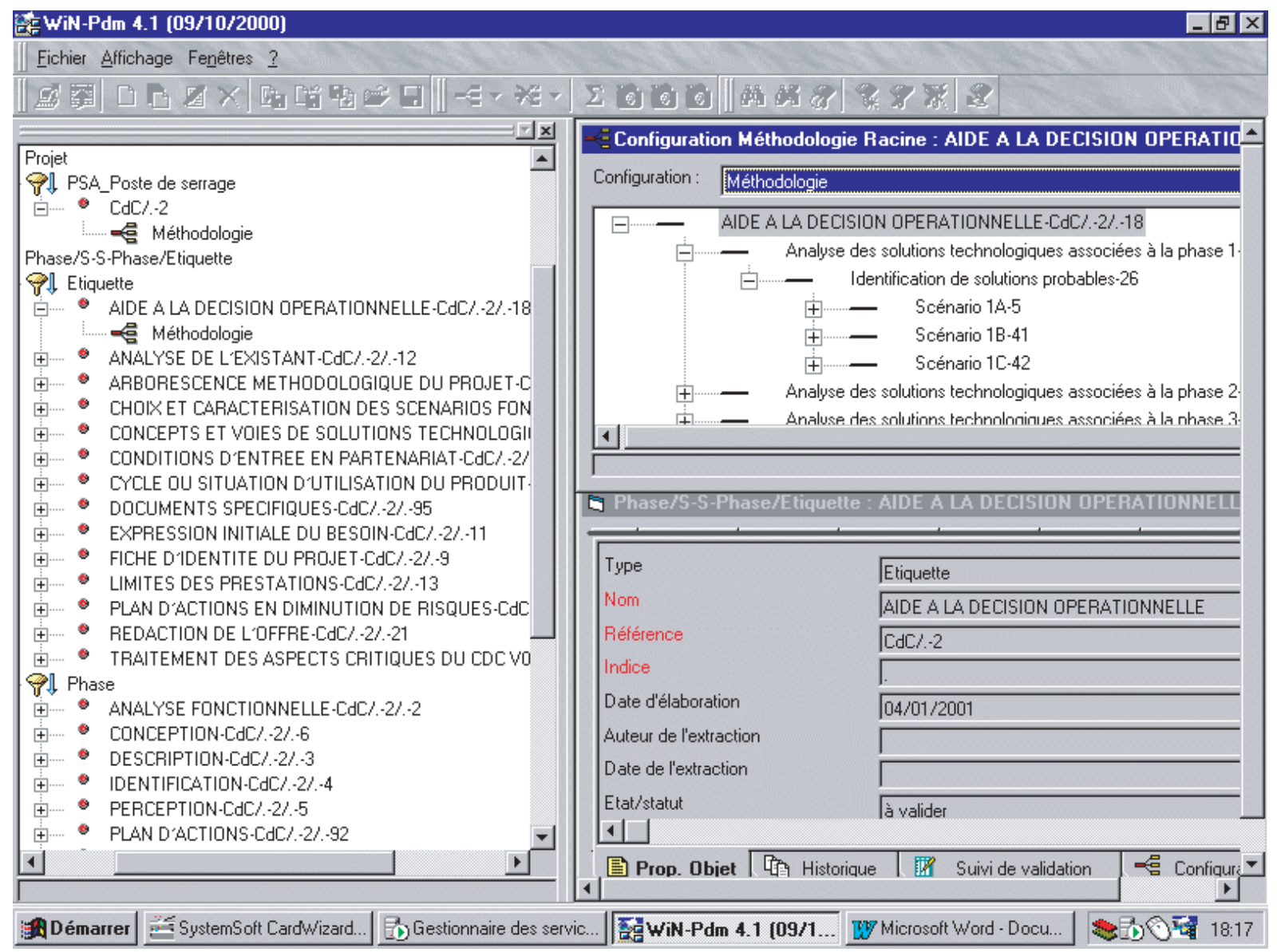

Fig. 5. Écran de la maquette informatique d'application de la méthodologie.

- de visualiser et d'archiver le reflet exact d'une configuration à un instant donné ;

- de produire et d'éditer de manière simple et rapide un état de la configuration réelle.

Elle permet au client d'exprimer son besoin de manière claire, concise et explicite, en intégrant dès le départ différents point de vues d'acteurs grâce à la constitution d'une équipe projet pluridisciplinaire. Véritable guide pour le chef de projet, elle permet ainsi l'élaboration d'un document structuré et riche en informations.

Ce sera sur ces bases que le fournisseur consulté pourra juger de sa capacité à répondre à l'appel d'offre. La méthodologie lui permet d'enrichir, compléter et traduire le besoin initialement exprimé tout en l'aidant à mettre en évidence, au travers d'une analyse des aspects critiques, les modes d'actions appropriés qui lui permettront de minimiser les risques. Elle devient donc pour le constructeur un véritable outil d'aide à la conception et à l'analyse des risques, ainsi qu'un indicateur favorisant la maîtrise des coûts et des délais. C'est à partir des données et des informations ainsi structurées que l'offre sera formalisée.

Le système de gestion de configuration permet au client d'analyser les différentes offres selon ses propres critères de comparaison et peut aisément hiérarchiser les fournisseurs selon l'intérêt de l'offre qu'ils ont émise.
La méthodologie proposée s'inscrit dans la démarche de l'analyse de la valeur et y apporte des ajouts : permet de revalider les buts et causes du projet, jalonne le projet afin de maîtriser les délais, la recherche de concepts et de solutions est facilitée grâce à une recherche par mots clés et l'appel à outils ajoute une étape d'analyse de risque. Elle permet d'élaborer des propositions novatrices et garantit la capitalisation des connaissances et du savoirfaire, plus généralement elle permet au constructeur d'assurer la maîtrise de ses informations. Elle s'inscrit dans le cadre de la gestion du processus de conception [10] mais appliquée à des produits complexes et unitaires.

La méthodologie constitue un support commun d'échange d'informations techniques aboutissant à la validation du cahier des charges de machine spéciale. Cette méthodologie peut être utilisée suivant 3 possibilités :

- par le client et le fournisseur s'ils possèdent tous les deux les outils :

- par le client ou le fournisseur si l'un ou l'autre possède l'outil (l'autre utilisant l'interface formulaire ou HTML).

La méthodologie s'appuie sur une analyse de terrain qui a permis de cerner les besoins industriels. Les donneurs d'ordre, comme les constructeurs à qui la démarche proposée a été présentée, ont montré un grand intérêt. 


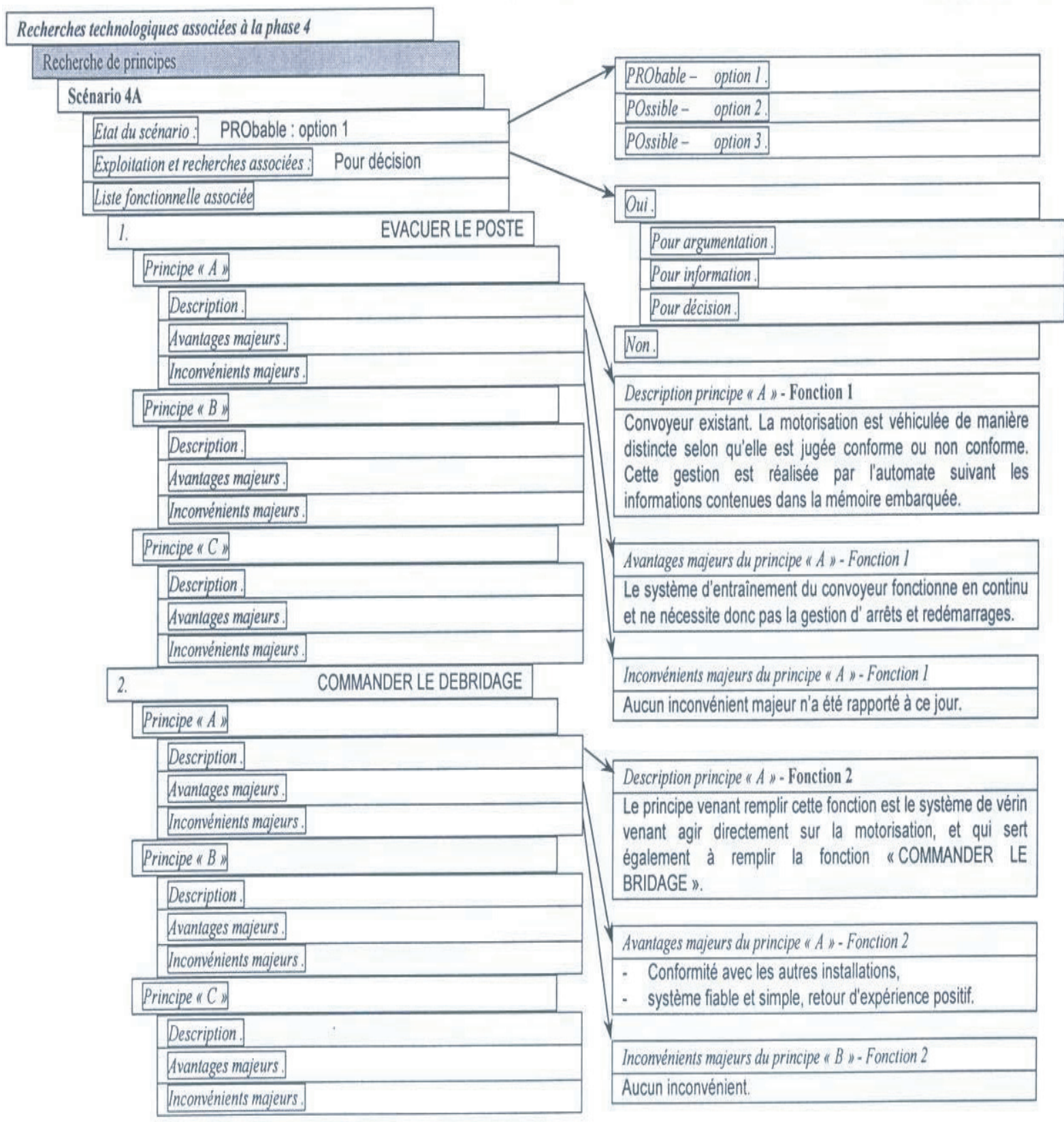

Fig. 6. Exemple d'arborescence de la métaphase 4 : aide à la décision opérationnelle. 
La validation à partir de documents clients a été réalisée sur un exemple de machine d'assemblage pour l'industrie automobile. Nous avons donc une démarche identifiée, ayant une rigueur très importante qui en cas de diversification de clientèle, va pouvoir sécuriser l'utilisateur face aux inconnues que présente un nouveau client ou projet ou donneur d'ordre. Les relations s'en trouvent donc améliorées.

La comparaison d'offres est possible en automatique à l'aide de l'outil permettant ainsi un échange plus rapide d'informations et donc des délais de validation de cahier des charges plus courts. La modélisation réalisée a permis la réalisation d'une application rapide de la méthodologie sur un progiciel de gestion de configuration du commerce. La gestion de configuration a pour objectif de fournir une vision claire de l'état d'une configuration (associée à un produit ou un projet) et de leurs évolutions en garantissant une traçabilité totale. Cette application étend le champ d'application des systèmes de gestion de données techniques [11] et de gestion de configuration.

Remerciements. Les auteurs tiennent à remercier les organismes qui ont participé au financement de cette étude : l'ANVAR LORRAINE, MECANICA, la région Lorraine et le FONGECIF de Lorraine (préparation du mémoire CNAM de L. Abt). Ce travail a été initié par M. Boissenot, ancien directeur général de MECANICA dont l'action soutenue pour le développement d'une activité de conception de machines spéciales en Lorraine doit être rappelée.

\section{Références}

[1] Conception de produits mécaniques, sous la direction de M. Tollenaere, Hermes, 1998
[2] L. Lossent, Contribution à la conduite d'étude de faisabilité de systèmes de fabrication, thèse de l'Université de Nancy 1, 1997

[3] P. Martin, J.D. Jeunehomme, Conception conjointe du produit et du système de fabrication. Approche à partir d'un exemple, Université d'Automne PRIMECA, Nancy, 20-22 octobre 1999

[4] O. Garro, Conception d'éléments physiques de systèmes de production - application aux machines outils à architecture parallèle, thèse de l'Université de Nancy 1, 1992

[5] AFNOR - NF X -50 151, Guide pour l'élaboration d'un cahier des charges fonctionnel

[6] L. Abt, Étude et formalisation d'une stratégie d'élaboration et de maîtrise du cahier des charges dédiée à la conception de machines spéciales, mémoire d'ingénieur CNAM, Nancy, 2001

[7] F. Limayem, B. Yannou, La méthode SPEC : une modélisation détaillée du Cahier des Charges Fonctionnel pour un suivi de projet et une maîtrise des risques, Congrès international AFAV 2000 : Les hommes et le management par la valeur, Paris, pp. 88-97

[8] C. Chevenier, B. Yannou, Maitrise des performances et des risques de projet - Enseignements d'application de SPEC à des cas industriels. Revue Française de Gestion Industrielle, Numéro spécial sur le Management par la Valeur, vol. 2, 2001

[9] B. Yannou, F. Limayem, La méthode SPEC : Suivi de Performances en cours de Conception. Third International Conference on Integrated Design and Manufacturing in Mechanical Engineering, Montréal, 2000

[10] A. Bernard, Modèles et approches pour la conception et la production intégrées, APII-JESA, 34/2000, pp. 163-193

[11] M. Tollenaere, Outils pour supporter l'information, Université d'Automne PRIMECA, Nancy, 1999

To access this journal online: www.edpsciences.org 